\title{
Flu vaccination campaign poses monitoring difficulties
}

$\mathrm{P}$ rovincial health authorities will have to shift into scramble mode this fall if they are to fully monitor the efficacy and safety of the pandemic (H1N1) 2009 vaccine as it will be administered near or during a resurgence of the virus, according to infectious diseases experts and public health officials.

"Some people who will be getting the vaccine will have H1N1 infections or will get infected shortly after getting the vaccine, so this will complicate matters," says Dr. Perry Kendall, provincial health officer for British Columbia. "Ideally, you would have the vaccine and deliver it before the virus or bacteria that you are trying to protect against is in the community."

Traditionally, seasonal flu hits Canada the hardest in late January or early February. The vaccine for seasonal flu is therefore administered in October or November, providing an adequate lead time to ensure everyone who wants to get the vaccine is immunized. To be effective, the vaccine must be received between five and seven days prior to exposure to the flu virus. People who require multiple shots should, ideally, wait two to four weeks between injections.

Canada is scheduled to receive 50 million doses of the vaccine from GlaxoSmithKline, though it won't be available until mid-November. No one is certain when a second wave of H1N1 flu might hit Canada, but some medical experts predict it will be earlier than the vaccine is available.

The timeline for manufacturing and administering the vaccine, however, will be compressed. This makes it more difficult to determine its efficacy (some people will already be incubating the virus when they receive the vaccine) and safety (there isn't enough time to enrol as many people in clinical trials as for a typical vaccine).

"We need to have a robust adverseevents surveillance system, beyond what we currently have, given the fact

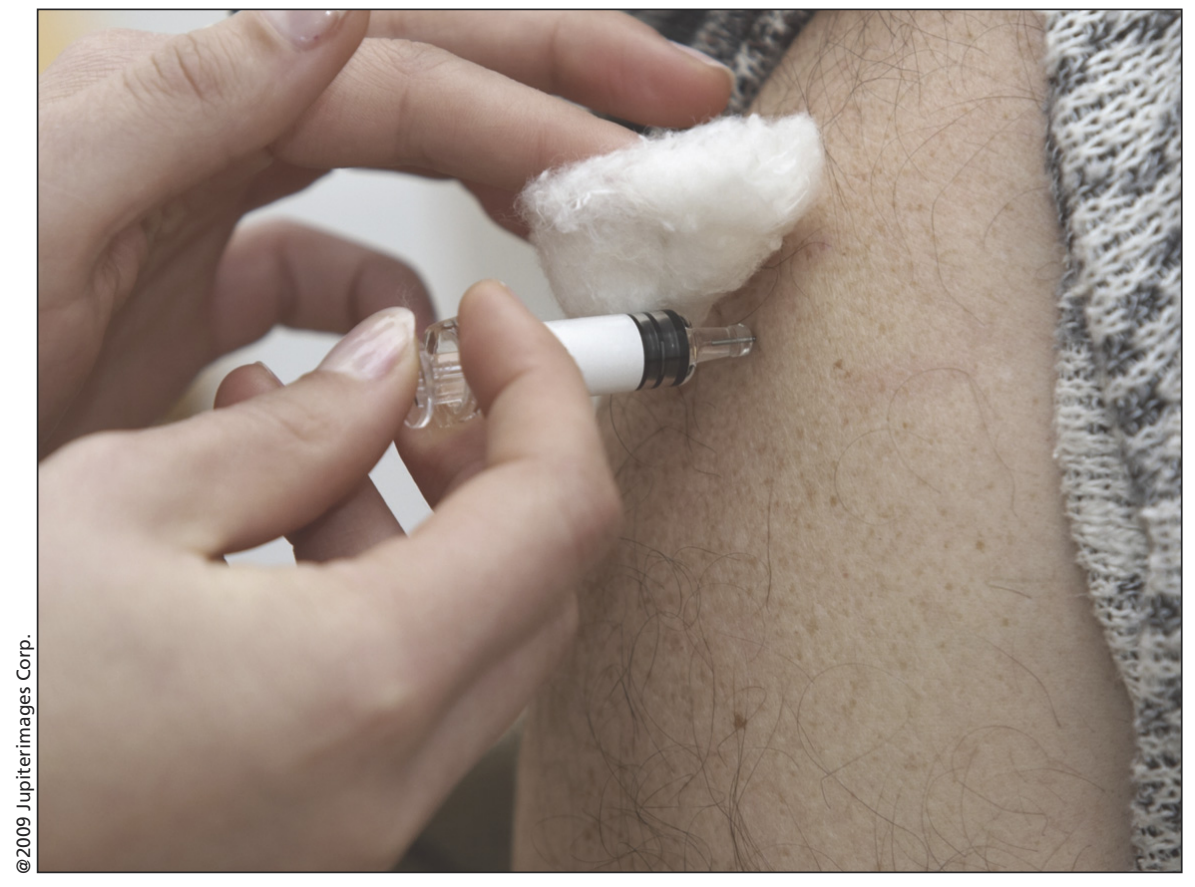

The unanswered question surrounding the pandemic (H1N1) flu vaccine is whether it will actually prevent illness.

that the timeline for clinical trials is short," says Dr. Robert Strang, chief public health officer for Nova Scotia. "And if you have either longer-term effects or rare events, you are not going to pick them up with the small number of people in a clinical trial."

Early results from one clinical trial underway on the $\mathrm{H} 1 \mathrm{~N} 1$ vaccine have shown it to be safe with no side effects. There is good reason to believe the vaccine will be safe and effective, says Dr. Gerald Evans, president of the Association of Medical Microbiology and Infectious Disease Canada. "We are going to presuppose that this vaccine is going to be just as effective and as safe as the seasonal flu vaccine because it is made in a similar fashion, with minor tweaking."

Still, because the vaccine is being rushed into production, some have raised concerns about its safety. They point to the 1976 swine flu vaccine campaign, which is believed to have caused an increase in cases of Guillain-
Barré syndrome, a neurological illness. It's estimated that the vaccine caused one extra case of the syndrome for every 100000 people who received it. In the United States, hundreds of people who were immunized acquired Guillain-Barré, and more than 30 of them died.

But the negative consequences of that vaccination program received excessive scrutiny, according to Kendall, because the 1976 swine flu pandemic failed to materialize. "Here we clearly have a pandemic, so the risk-benefit calculation is completely different than in 1976," he says. "Millions of people were vaccinated against a virus that didn't circulate in the community. There was no benefit, only downsides."

Like Strang, Kendall says that postmarketing surveillance of the H1N1 vaccine will be very important. A national expert group has been established to determine how it should be done. 
But health officials in Ontario express confidence that the province will be able to effectively monitor the vaccine's safety. "Ontario is experienced in monitoring and reporting adverse events all year long when many vaccines are administered to the public and is expected to effectively monitor any adverse event reported during both the seasonal influenza vaccine program and the $\mathrm{H} 1 \mathrm{~N} 1$ vaccine program," David Jensen, a media relations coordinator with Ontario's Min- istry of Health and Long-Term Care, writes in an email. "Canada is a world leader in monitoring and reporting any unusual post-vaccine events."

As for determining efficacy, that will be no easy task, given that a large number of people (up to 25 million Canadians, by some estimates) will be receiving the vaccine very quickly (perhaps, at a rate of 3.5 million doses a week) and may be receiving it during a pandemic (H1N1) 2009 outbreak, according to Canada's Chief Public Health Officer,
Dr. David Butler-Jones. As such, a surrogate marker might be the best means to determine how well the vaccine works.

"The real thing we would like to know is: Does it prevent illness?" says Evans. "All we are going to be able to figure out is that the people who took the vaccine have a measurable amount of antibodies, which will correlate in a reduction of the likelihood of getting the illness." - Roger Collier, CMAJ

DOI:10.1503/cmaj.109-3044 